\title{
Salvatore D'ONOFRIO, Le Sauvage et son double
}

\author{
Nicole Belmont
}

\section{(2) OpenEdition}

\section{Journals}

\section{Édition électronique}

URL : https://journals.openedition.org/clo/1372

DOI : $10.4000 /$ clo. 1372

ISSN : 2266-1816

Éditeur

INALCO

\section{Édition imprimée}

Date de publication : 30 décembre 2011

Pagination : $175-177$

ISBN : 978-2-85831-202-3

ISSN : 0396-891X

\section{Référence électronique}

Nicole Belmont, «Salvatore D'Onofrio, Le Sauvage et son double », Cahiers de littérature orale [En ligne], 70 | 2011, mis en ligne le 18 mars 2013, consulté le 02 juillet 2021. URL : http:// journals.openedition.org/clo/1372 ; DOI : https://doi.org/10.4000/clo.1372

Ce document a été généré automatiquement le 2 juillet 2021.

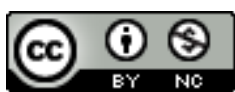

Cahiers de littérature orale est mis à disposition selon les termes de la Licence Creative Commons Attribution - Pas d'Utilisation Commerciale 4.0 International. 


\title{
Salvatore D'ONOFRIO, Le Sauvage et son double
}

\author{
Nicole Belmont
}

\section{RÉFÉRENCE}

Salvatore D'ONofRIo, Le Sauvage et son double, Paris, Les Belles Lettres, 2011, 268 p., coll. «La Vérité des mythes ». ISBN : 978-2-251-98560-0.

1 Dense quant aux matériaux qu'il met en œuvre, complexe quant aux questions théoriques qu'il soulève, cet ouvrage s'attache aux figures de doubles dans la littérature mythique, en allant bien au-delà d'une simple opposition entre civilisation et sauvagerie. Ne serait-ce qu'en raison de « l'impossibilité de penser l'un des deux termes de l'opposition sans penser l'autre » comme l'affirme Philippe Descola (cité par l'auteur p. 22).

2 Littérature mythique, qu'est-ce à dire ? Il s'agit de textes qui nous sont parvenus par l'écrit, qui émanent de cultures anciennes comme l'Épopée de Gilgamesh, « l'histoire la plus ancienne du monde»(p.31), de l'Odyssée (Ulysse et Polyphème), de récits bibliques (Caïn et Abel, Jacob et Esaü, Jésus et Jean-Baptiste), de l'Occident médiéval avec le Roman de Renart, ou la Chanson de Roland, que Salvatore D'Onofrio n'hésite pas à aborder par le biais du théâtre de marionnettes sicilien qui perdure encore. Il est certain que toute cette matière littéraire qui nous est parvenue sous forme écrite s'origine dans la transmission orale. Sa cristallisation scripturale nous prive de variantes et de versions dont ne peuvent témoigner qu'un petit nombre de transcriptions présentant des différences entre elles. Il est rare, et d'autant plus méritoire que ces textes soient étudiés dans leur qualité mythique à la façon dont les anthropologues approchent les matériaux recueillis dans des cultures exotiques censées procéder de la " pensée sauvage ». Pour l'auteur, relèvent du mythe dans cette littérature la redondance, le double niveau de signification et la présence d'énigmes. Du côté du traitement analytique de ces textes, j'ajouterais qu'ils exigent l'attention au 
moindre détail, en toute bonne méthode structuraliste. Je citerai un passage du chapitre qui concerne "Ulysse et l'homme sauvage ». Ulysse, épuisé, aborde enfin, mais sans le savoir sur sa terre. Il choisit de se mettre à l'abri d'un buisson et se glisse « sous un berceau d'oliviers emmêlés : l'un était greffé, l'autre franc ».

S'entrelacent ici bien des représentations du sauvage et du domestique, le héros étant presque comme dans une tanière ou dans la phase liminale d'un rite de passage, pour lui permettre par des symboles ambivalents de traverser le seuil audelà duquel récupérer, à son retour chez lui, sa pleine humanité (p. 83).

Dans les textes mythiques, tout est signifiant, alors qu'une approche superficielle ignore ou stigmatise comme non pertinents, voire absurdes certains motifs ou épisodes. La densité et la complexité de cet ouvrage tiennent à ce que l'auteur se donne pour tâche de démontrer la cohérence implacable de ces textes à travers tous leurs moindres éléments.

4 Le fil conducteur est donc la figure du double, plus exactement les figures du double puisqu'elles sont toutes porteuses de différences entre elles ${ }^{1}$. Leur proximité, leurs similitudes ne servent qu'à signaler et/ou produire l'altérité, c'est-à-dire l'identité de chacun. Le chapitre III, "Les doubles et la Bible», s'attachant principalement aux figures du Christ et de son double, le sauvage Jean-Baptiste ${ }^{2}$, démontre une érudition confondante. L'auteur reconstitue des arbres généalogiques où il repère des incestes du deuxième type, autre mode de redoublement caché. Il termine ce chapitre dense par cette précieuse remarque.

L'histoire de Jean-Baptiste et du Christ non seulement montre l'importance des relations parentales dans les récits mythiques de la Méditerranée, notamment dans les histoires de double (comme nous l'avons déjà vu pour Abel et Caïn, Jacob et Esaü ou Romulus et Remus), mais elle apporte en plus un éclairage pertinent sur la construction de la parenté et du mythe selon une même logique de manipulation symbolique (p. 148).

5 À propos du chapitre IV, «Renart et son compère ", j'aimerais signaler avant de conclure, un rapprochement que je soumets à la sagacité de S. D'Onofrio. Dans le Roman de Renart, textes médiévaux largement étudiés, les deux personnages principaux, Ysengrin, le loup, et Renart, le goupil, sont dits compères entre eux. Le compérage est une parenté spirituelle qui découle du baptême. Dans les contes d'animaux d'origine orale collectés à partir du xix siècle, ils sont également des doubles, s'opposant l'un à l'autre dans des aventures où le renard gagne le plus souvent. Dans une de ces aventures, recueillies en un grand nombre de versions, il invoque à trois reprises le fait qu'il est appelé comme parrain ${ }^{3}$ : en réalité pour aller manger de la nourriture qui avait été mise de côté par les deux compères (doublement compères). Lorsqu'il revient auprès du loup, il énonce les noms de ses filleuls successifs qui sont une allusion à son forfait, ce que le loup ne comprend pas : «Entamé », « Moitié » et "Bien-léché », dans cette même version. Persiste donc, dans ces récits d'origine entièrement orale, cette idée de compérage : réminiscence des textes médiévaux ou manière propre à la pensée « sauvage » occidentale d'exprimer l'idée de double?

6 J'aimerais citer pour terminer un passage de la conclusion de S. D'Onofrio avec lequel je me sens en complet accord.

Ces dédoublements structuraux soulignent davantage la tendance des textes littéraires mythiques à " aveugler » celui qui les lit ou les écoute, pour l'empêcher de saisir leur signification profonde. [...] Cependant, les vérités ultimes qu'un auteur confie dans son histoire ne doivent pas rester indéchiffrables à jamais. La manière la plus féconde d'aborder les énigmes a été sans doute la plus simple : rapprocher 
les textes - les personnages, leurs actions et qualifications - dans un modèle capable de les faire parler entre eux (p. 212).

On ne peut mieux dire.

\section{NOTES}

1. Les « vrais » jumeaux n'existent pas, en dépit de l'expression répandue, nous apprenait il y a peu un article publié dans Le Monde Science et technologie du 6/12/2012.

2. "double presque gémellaire, par ventres séparés, pourrait-on dire » (p. 142).

3. «- Ah je crois qu'on m'appelle pour être parrain. - Ma foi, qu'il lui dit, i te faudrait y aller, va, on ne refuse pas le baptême » (version du Doubs). 